\title{
TES in optic neuropathies and RP
}

\author{
Umur Kayabasi*1 \\ ${ }^{1}$ Liv Hospital, Istinye University, Istanbul
}

\begin{abstract}
Objective: Aim was to achieve improvement in vision and visual fields in optic neuropathies and retinitis pigmentosa (RP) by transcorneal electrical stimulation (TES).

Methods: 20 patients with non-arteritic anterior ischemic optic neuropathy, 10 patients with traumatic optic neuropathy and 10 with RP were stimulated using different protocols for optic neuropathies and RP. Patients with optic neuropathy were treated by TES 2 months after the acute event.
\end{abstract}

Results: In all groups, improvement both in vision and visual fields were achieved. The average visual acuity improvement in all groups was 2 Snellen lines. Visual field improvements after 10 days of TES were documented. Changes before and after treatment in Mean Deviation was statistically significant $(\mathrm{P}<0,001)$.

Conclusion: TES may be considered as a safe and effective treatment in certain optic neuropathies and RP.

\section{Introduction}

Transcorneal electrical stimulation (TES) is an accepted treatment modality in retinitis pigmentosa (RP) [1]. Electrical stimulation may be used to induce neuroprotective growth factors in the retina. The main aim of TES has been declared as to stabilize vision and to stop visual field (VF) deteriorization in this progressive disease [2]. The results of some multicenter studies have been encouraging and patients have been instructed to use TES themselves at their home settings [3]. This treatment modality is continued for about a year and patients are periodically examined by their physicians. There are also ongoing clinical trials about this therapy in non-arteritic anterior ischemic optic neuropathy (NA_AION) and traumatic optic neuropathy (TON) [4]. Another study performed for 10 days using lid electrodes and transorbital stimulation instead of transcorneal impulses in optic neuropathies resulted in an average of $25 \%$ improvement in VF defects [5]. In this study, alternating current therapy was applied through the eyelids. In the treatment of optic neuropathies TES may be as effective as transorbital way since stimulation may give more benefit when passing through a liquid environment (Figure 1).

\section{Methods}

TES therapy was performed in 20 NA_AION, 10 TON and 10 $\mathrm{RP}$ patients. Okuvision $\mathrm{GmbH}$ device was used for the therapy. Transcorneal stimulations were done for 40 minutes per day in 10 consecutive days in patients with optic neuropathies whereas in RP TES was performed for 30 minutes per week for 6 months. The phosphene threshold was detected in each patient and $200 \%$ of this level was applied. The stimulations were performed at least 2 months after the initial visual loss in optic neuropathies. The age range was between 19 and 66. In patients with optic neuropathy, the involved eye was stimulated; in RP, both eyes were stimulated.

\section{Results}

In 4 patients with NA_AION, visual acuity improvement more than 3 Snellen lines was observed. In 10 patients, improvement in visual

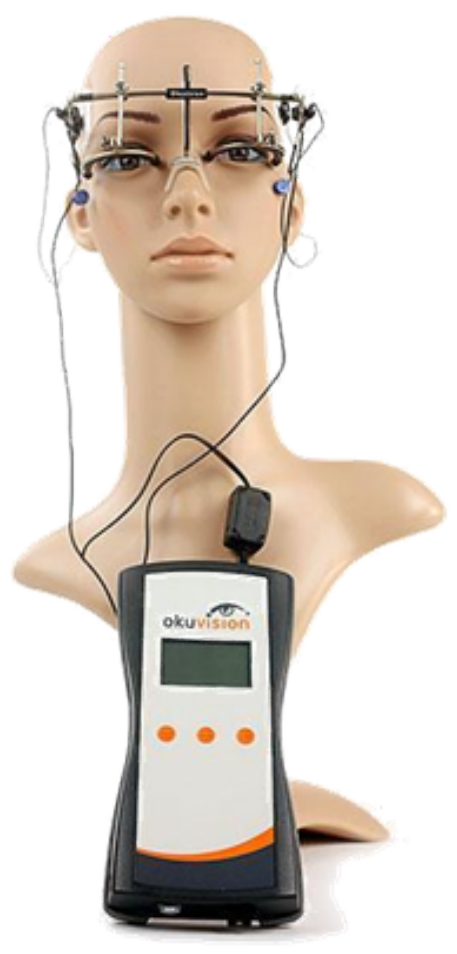

Figure 1. TES

Correspondence to: Umur A. Kayabasi, MD, Assistant ProfessorNeuroophthalmology, Liv Hospital- Istinye University, Istanbul, Tel: 90532 6129050; E-mail: kayabasi@yahoo.com

Key words: TES, optic neuropathy, RP, visual fields

Received: March 03, 2017; Accepted: March 14, 2017; Published: March 17, 2017 
acuity was between 1 and 3 Snellen lines. 6 patients were stable, but reported increase in brightness and color sensations. Ischihara color plate examination revealed more than 2 plates improvement in 3 of these patients. VF examination disclosed improvement in 10 patients (Figure 2). In TON group, visual acuity improvement more than 3 Snellen lines was achieved in 3 patients. In 4 patients improvement was between 1 and 3 lines. In the other 3 patients, no change was observed.
VF expansion more than 10 degrees was detected in 4 patients (Figure 3 ). In the RP group, visual acuity improved more than 3 Snellen lines in 3 patients and in 2 patient's improvement was between 1 and 3 lines (Table 1). No adverse effects were recorded. As a whole, average improvement of VA by 2 lines was achieved. Improvement in VF was investigated by calculating the change in Mean Deviation (MD) before and after stimulations in all the groups

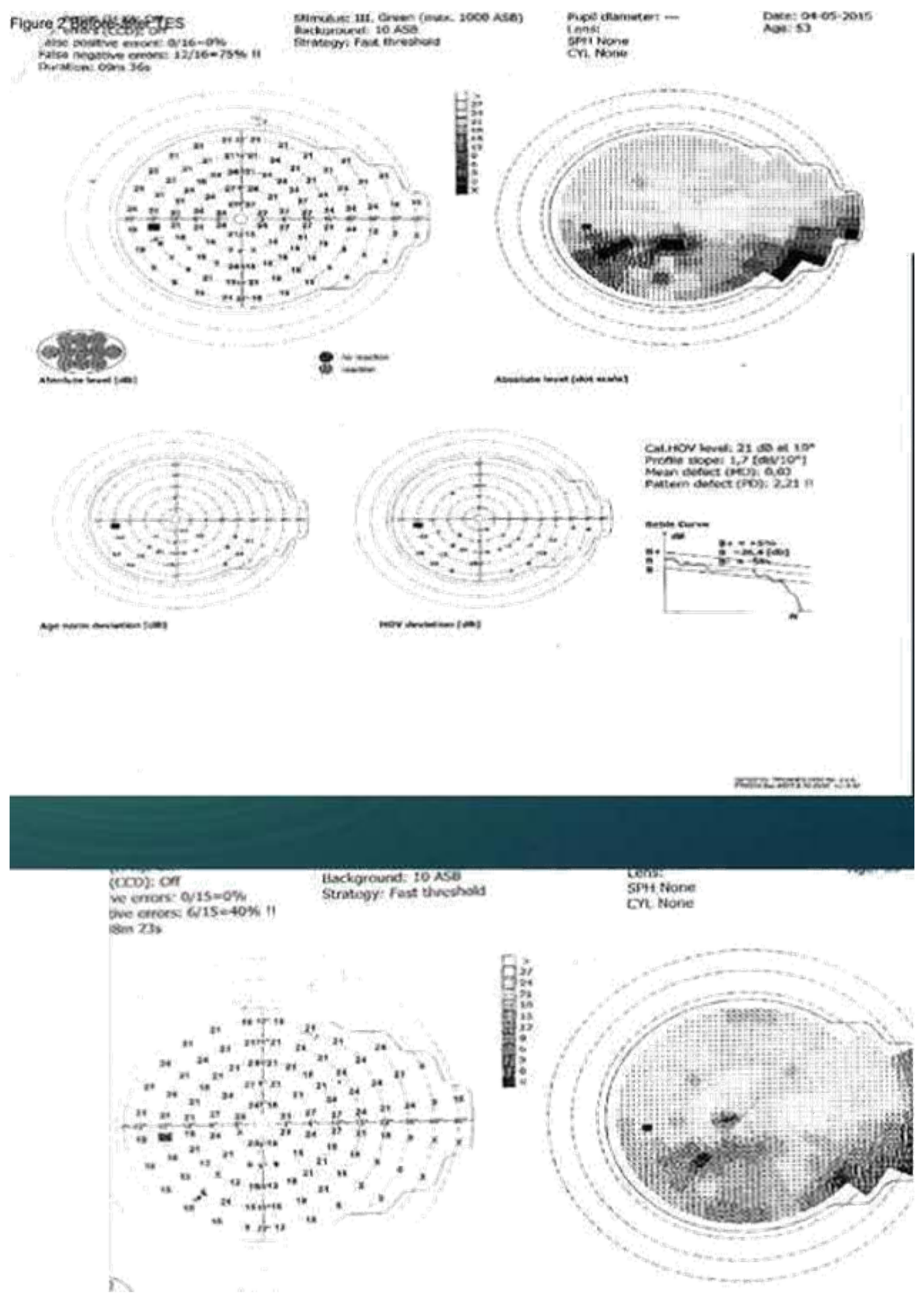

Figure 2. Before and after TES in AION. 


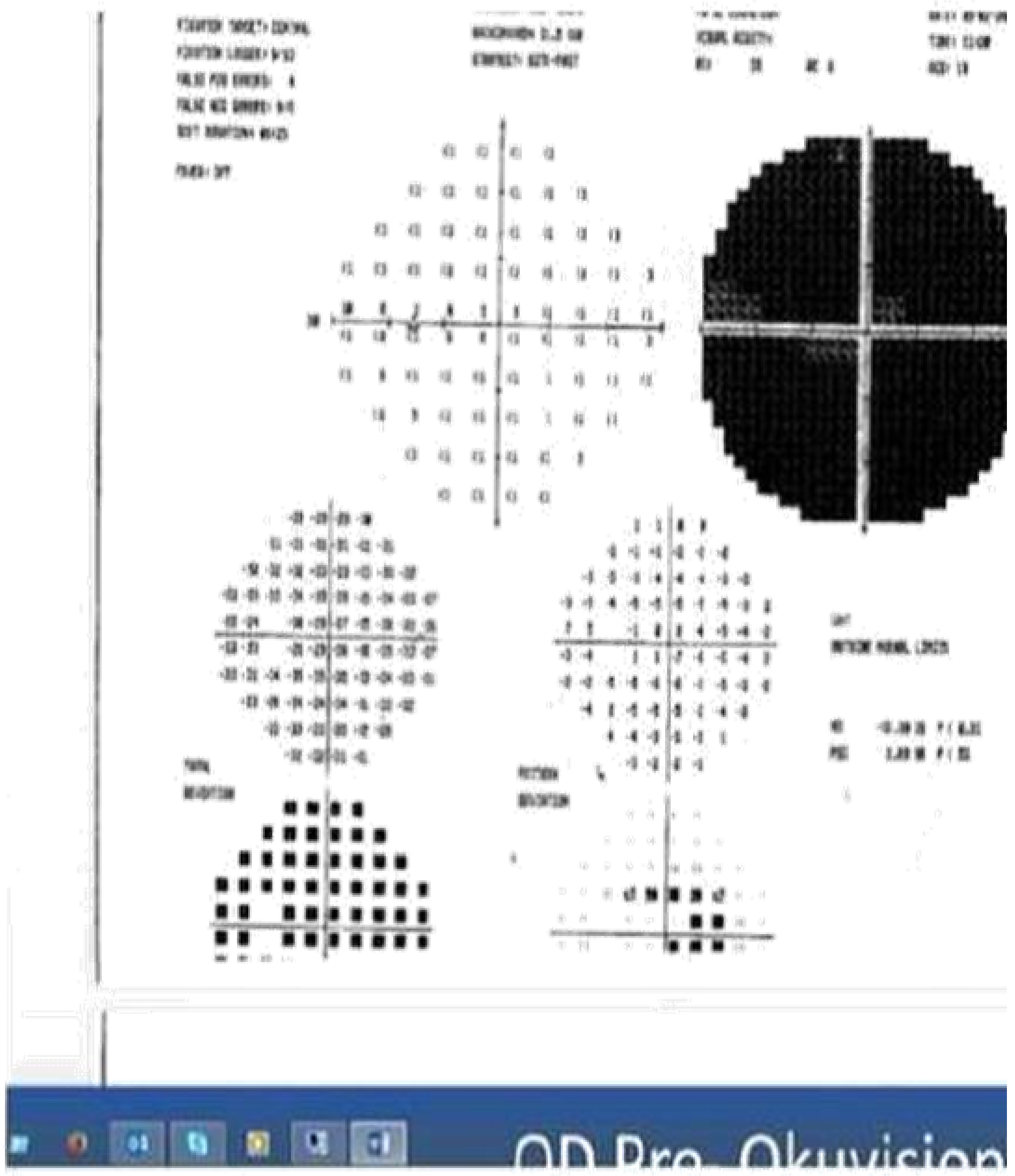

Figure 3. Before TES in TON.

Table 1. TES results.

\begin{tabular}{|c|c|c|c|}
\hline & AION & TON & RP \\
\hline VA $>3$ Line & 4 patients & 3 patients & 4 patients \\
\hline $1<$ VA $<3$ & 10 pat.s & 3 patients \\
\hline STABLE & 6 pat.s & 5 patients \\
\hline
\end{tabular}


Average improvemnent in MD was statistically significant $(\mathrm{P} \leq$ $0,001)$.

\section{Discussion}

TES, by stimulating the retina of the patients (retino-fugal stimulation), induces a series of action potentials that travel on the optic nerve back into the visual center of the brain (Figure 4, 5 and 6). The brain interprets these signals as light sensations (phosphenes). The TES device was designed to promote cell regeneration in photoreceptors and delay progressive sight loss by sending small amounts of electrical current to stimulate the retina, optic nerve and pathways in the brain. The results of a pilot study in 2011 demonstrated that patients with RP receiving stimulation showed a statistically significant improvement in their field of vision [1]. The results of our study suggest that TES is able to stimulate some inactive cells on the optic nerve and the electrical current has the capability of making these cells active again. Between the dead neurons and healthy cells in partial optic nerve damage, there may be some inactive cells in the transition zone which can be reactivated by micro electrical current [6]. The improvement in patients with VF defects may also suggest that the stimulation is effective in the visual pathways in the brain, too. Some of the patients with NA_AION and TON in our study showed marked improvement in VF as demonstrated by the computerized visual field testing. There have been some prior publications about improvements in optic neuropathies after TES, but our study is the first that reports improvement both in optic neuropathies and retinal degenerations after stimulations. The optimal

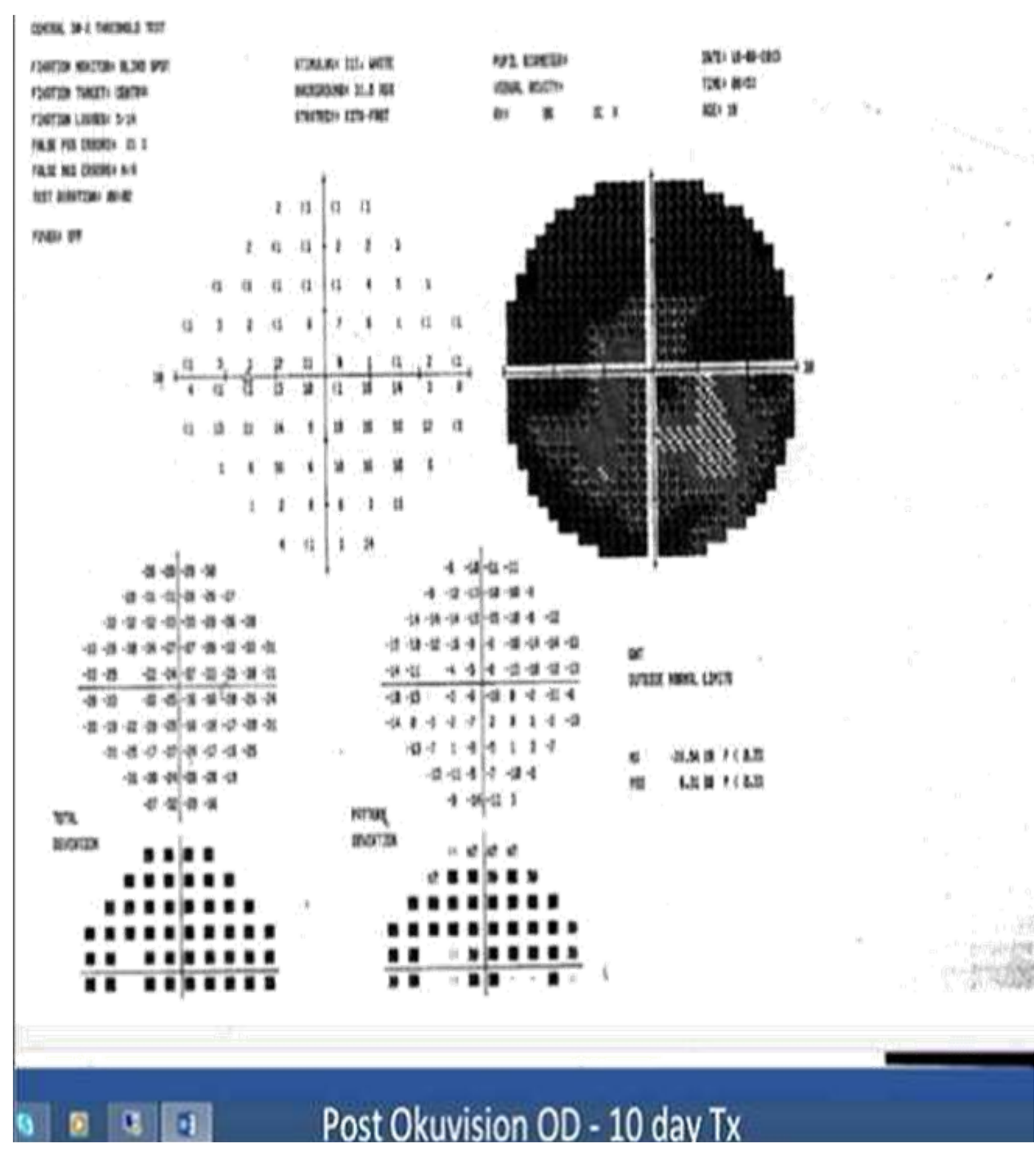

Figure 4. After TES in TON. 


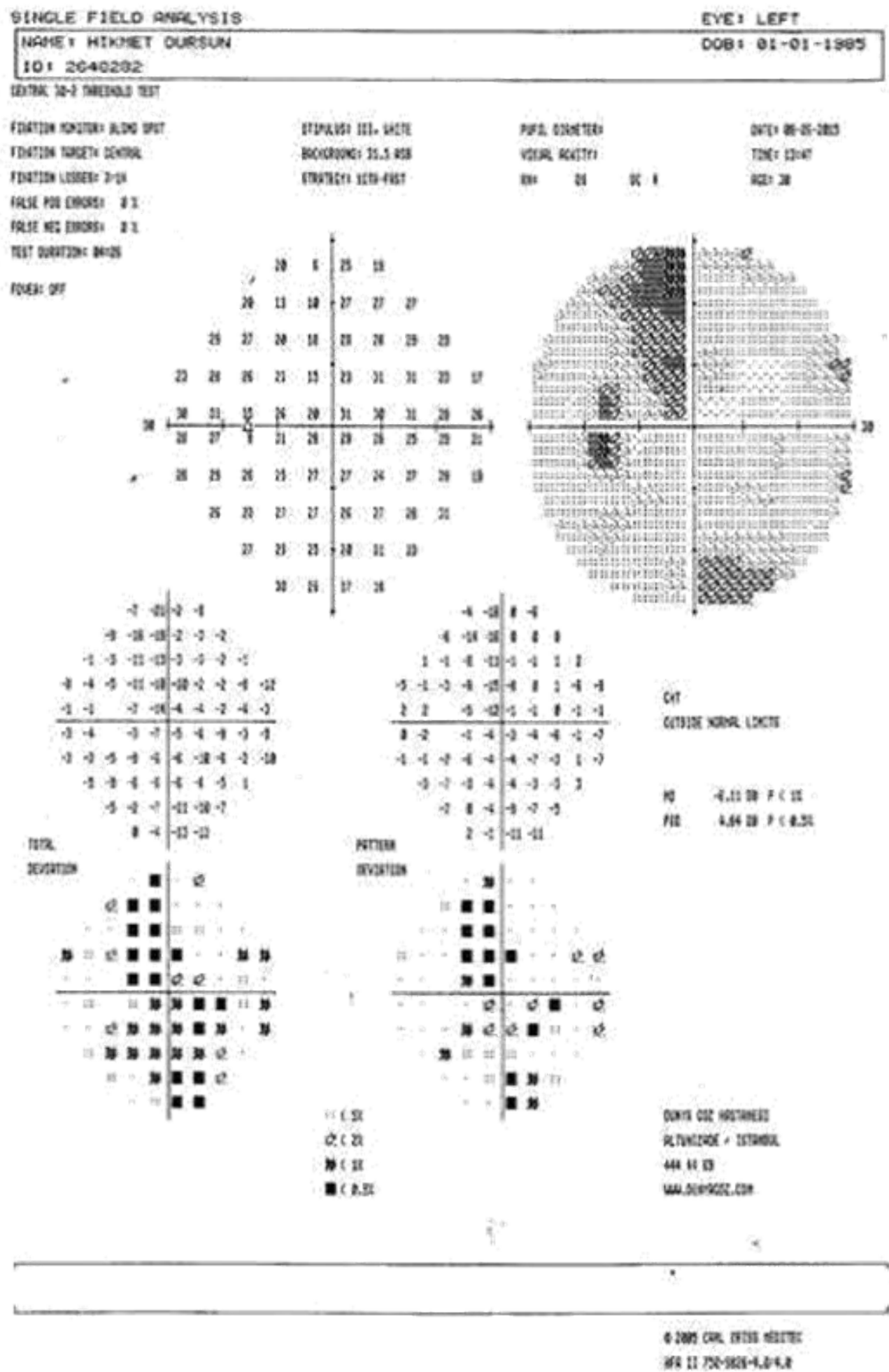

Figure 5. Before TES in TON. 


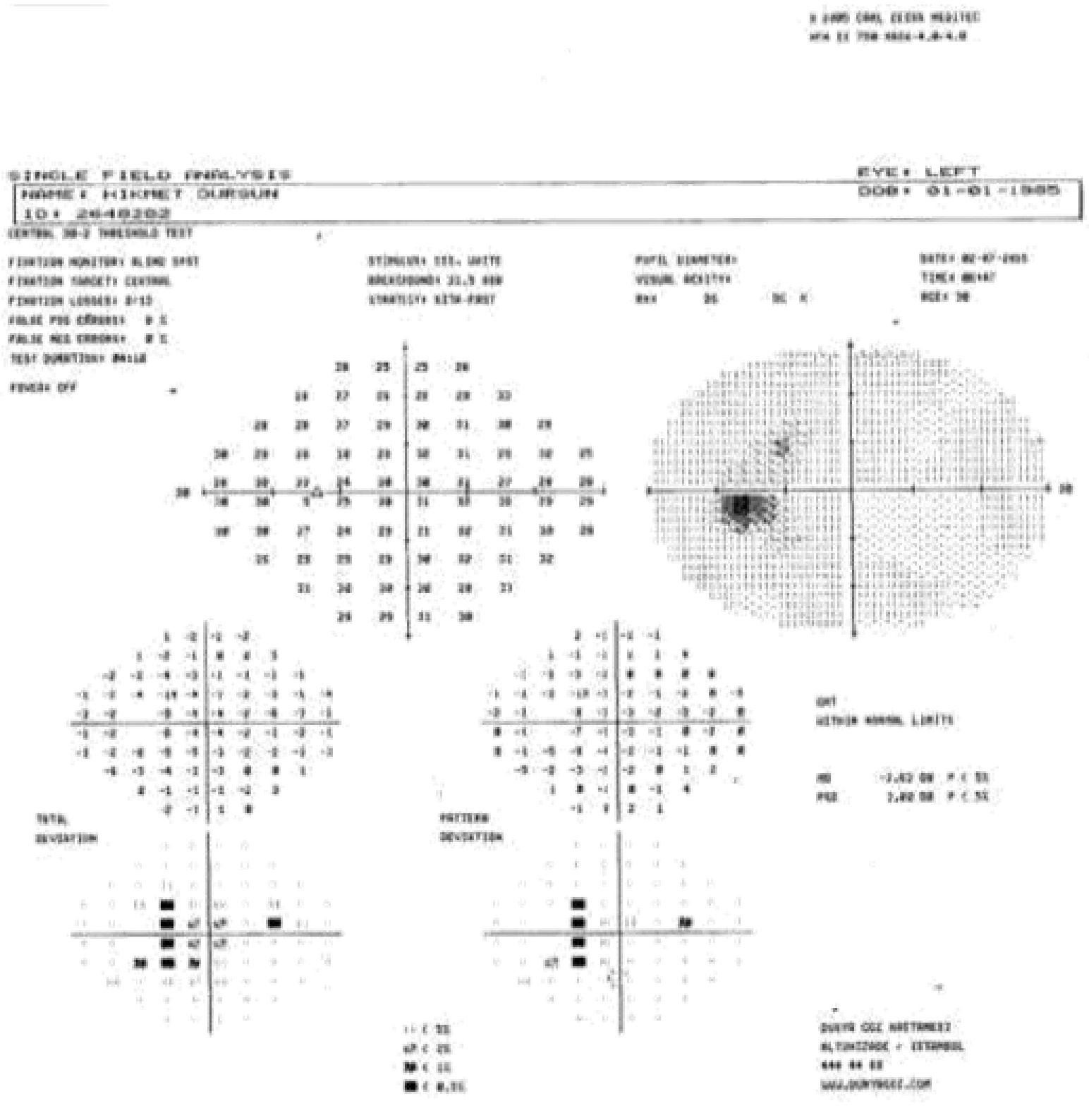

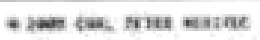

Figure 6. After TES in TON.

duration of TES in NA_AION, TON and RP is open to debate and there is the possibility of further improvement with the continuation or repetition of stimulations after a certain amount of time. Current recommendation in RP has been persistent stimulation because of some observations regarding deterioration of vision after finishing the therapy. Further studies will be necessary to resolve these issues.

\section{Conclusion}

Our results which disclose improvements but in visual acuity and VF may suggest that TES is a safe and effective treatment modality in certain optic neuropathies and RP.

\section{References}

1. Schatz A, Röck T, Naycheva L, Willmann G, Wilhelm B, et al. (2011) Transcorneal electrical stimulation for patients with retinitis pigmentosa: A prospective, randomized, sham-controlled exploratory study. Invest Ophthalmol Vis Sci 52: 4485-4496. [Crossref]

2. Naycheva L, Schatz A, Röck T, Willmann G, Messias A, et al. (2012) Phosphene thresholds elicited by transcorneal electrical stimulation in healthy subjects and patients with retinal diseases. Invest Ophthalmol Vis Sci 53: 7440-7448. [Crossref]

3. Wang ET, Zhao M (2010) Regulation of tissue repair and regeneration by electric fields. Chin J Traumatol 13: 55-61. [Crossref]

4. Brian Burke (2015) Electric Stimulation of the Eye to Improve Vision After Trauma. Wills Eye. Clinicaltrials.gov. 
5. Bola M, Gall C, Moewes C, Fedorov A, Hinrichs H, et al. (2014) Brain functional connectivity network breakdown and restoration in blindness. Neurology 83: 542-551. [Crossref]
6. Gall C, Schmidt S, Schittkowski MP, Antal A, Ambrus GG, et al. (2016) Alternating Current Stimulation for Vision Restoration after Optic Nerve Damage: A Randomized Clinical Trial. PLoS One 11: e0156134. [Crossref]

Copyright: $\odot 2017$ Kayabasi U. This is an open-access article distributed under the terms of the Creative Commons Attribution License, which permits unrestricted use, distribution, and reproduction in any medium, provided the original author and source are credited. 